\title{
The ionising cluster of 30 Doradus ${ }^{\star}$
}

\section{Stellar kinematics}

\author{
G. Bosch ${ }^{1,2}$, F. Selman ${ }^{3}$, J. Melnick ${ }^{3}$, and R. Terlevich ${ }^{1, \star \star}$ \\ 1 Institute of Astronomy, Madingley Road, Cambridge CB3 0HA, UK \\ 2 Facultad de Ciencias Astronómicas y Geofísica, La Plata, Argentina \\ 3 European Southern Observatory, Alonso de Córdova 3107, Santiago, Chile
}

Received 6 June 2001 / Accepted 4 October 2001

\begin{abstract}
On the basis of multislit spectroscopy of 180 stars in the ionising cluster of 30 Doradus we present reliable radial velocities for 55 stars. We calculate a radial velocity dispersion of $\sim 35 \mathrm{~km} \mathrm{~s}^{-1}$ for the cluster and we analyse the possible influence of spectroscopic binaries in this rather large velocity dispersion. We use numerical simulations to show that the observations are consistent with the hypothesis that all the stars in the cluster are binaries, and the total mass of the cluster is $\sim 5 \times 10^{5} M_{\odot}$. A simple test shows only marginal evidence for dynamical mass segregation which if present is most likely not due to dynamical relaxation.
\end{abstract}

Key words. stars: early-type - stars: kinematics - binaries: spectroscopic - galaxies: clusters: general galaxies: Magellanic Clouds

\section{Introduction}

A significant fraction of the old stars we now observe in galaxies appear to have formed in Starbursts. Therefore, understanding violent star formation becomes crucial if we want to understand the star formation history of the Universe. 30 Doradus in the LMC is the nearest and best studied example of a massive starburst cluster and thus it has become a sort of "Rosetta Stone" for deciphering the physics of violent star formation processes (Walborn 1991; Selman et al. 1999b (Paper III) and references therein).

Although 30 Dor has been the subject of intensive observational effort from the ground and space there are still a number of critical problems that remain unsolved. Perhaps the most burning open problem is the issue of mass segregation first raised by Malumuth \& Heap (1995) that has important implications for our understanding of the process of cluster formation in general (Clarke 2001).

This Paper is the fourth of a series devoted to a comprehensive study of the 30 Doradus starburst cluster (NGC 2070). We can summarize the central results of the previous papers of this series as follows:

- The Initial Mass Function (IMF) of NGC 2070 between 3 and $120 M_{\odot}$ is very well represented by a Salpeter

Send offprint requests to: G. Bosch, e-mail: guille@f caglp.unlp.edu.ar

* Based on observations collected at the European Southern Observatory.

** Visiting Professor, INAOE, México. power-law. The total "photometric" mass of the cluster - inside $20 \mathrm{pc}$ - from $0.1 M_{\odot}$ to $120 M_{\odot}$ is $M_{\text {phot }} \sim$ $3 \times 10^{5} M_{\odot}$;

- The star-formation history of the cluster shows three distinct peaks centered 5 Myrs, 2.5 Myrs, and $<1.5$ Myrs ago with no evidence for a spatial segregation of ages;

- The density distribution of stars between 20 and $60 M_{\odot}$ is a power-law of slope of -2.85 . This means that the total mass increases very slowly with radius $\left(r^{0.15}\right.$ or even logarithmically);

- There is some evidence for mass segregation revealed by the fact that the most massive stars appear to be more concentrated toward the center. However, there is a distinct "ring" of massive stars located at about 6 pc from the center of the cluster (R136). This ring contains stars of the three age groups described above.

The purpose of the present Paper is to study the stellar kinematics of the brightest stars in the cluster using the NTT observations described in Paper II of this series (Bosch et al. 1999). We use the data to investigate the presence of dynamical mass segregation in the cluster, and to attempt to constrain the lower end of the IMF from the comparison between the photometric and the dynamical masses.

\section{Observations and data reduction}

The observations - already described in Paper II were obtained with the La Silla NTT telescope using 
the Multislit option of EMMI's RILD mode. In this mode, grism \# 5 was used, which in combination with a 0.8 arcsec wide slit yields a resolution of $1.3 \AA /$ pixel. The wavelength range is 3600 to $6000 \AA$ in most cases, although the limits vary slightly between individual spectra, as they depend on the position of each slitlet within the mask. A total of 7 masks were produced each including an average of 35 slitlets. The basic reduction steps were described in Paper II including bias correction, flat fielding, wavelength calibration, and aperture extraction. Here we need to discuss some aspects of the wavelength calibration not included in Paper II that are particularly relevant for the determination of radial velocities.

During the observations a technical problem made the Argon lamp too weak to produce reliable identification lines for 2 masks while the strong He I $5875 \AA$ arc line is saturated for several spectra. The problem is worst when both effects appear at the same time as there are no reliable comparison lines redwards He I $5015 \AA$. For example, this makes useless the He II $5411 \AA$ absorption line for radial velocity measurements in the affected spectra.

The problems with the calibration lamps also altered the normal sequence in which the lamps were taken; the calibration exposures for the last three masks had to be taken at the end of the night. Although there were no telescope presets, the telescope was still tracking so the position angle of the instrument rotator slowly changed and this may introduce systematic errors. We address this issue in the following section.

\section{Radial velocities}

\subsection{Zero-point errors}

The problems with the calibration lamps may introduce systematic differences between the zero points of each mask. We have used the radial velocity of the [O I] $5577 \AA$ Auroral line, whenever possible, to test for this effect.

The straightforward approach would be to add algebraically the radial velocity of the sky line to the velocity of each star. Unfortunately, however, the problems with the calibration lamps described above imply that several spectra have unreliable wavelength calibration in the region of $[\mathrm{O} \mathrm{I}]$.

For most spectra the formal (measurement) error in the position of the sky line is $\sim 6.6 \mathrm{~km} \mathrm{~s}^{-1}$. The mean radial velocity of the $[\mathrm{O} \mathrm{I}]$ lines with reliable calibrations is $-6.5 \mathrm{~km} \mathrm{~s}^{-1}$ with a dispersion of $12.9 \mathrm{~km} \mathrm{~s}^{-1}$. Thus, while we cannot correct the individual velocities for systematic zero point errors, the radial velocity dispersion must be corrected by this effect by subtracting quadratically the $\left[\mathrm{O}\right.$ I] dispersion $\sigma_{[\mathrm{O} \mathrm{I}]}=\sqrt{12.9^{2}-6.6^{2}}=10.9 \mathrm{~km} \mathrm{~s}^{-1}$.

\subsection{Velocity determination}

As mentioned above, the resolution of our combination of dispersion grating and camera yields $1.3 \AA$ per pixel. For 2-pixel sampling this corresponds to a spectral resolution of about $165 \mathrm{~km} \mathrm{~s}^{-1}$ at $4750 \AA$. The ultimate limit attainable in the precision of Doppler shifts is dominated by the photon noise in the spectrum (Brown 1990). The uncertainty in the measured radial velocity for the case of a single line of width $w$ and depth $d$, measured in units of the continuum intensity $I_{\mathrm{c}}$, is given by

$\delta v_{\mathrm{rms}}=\frac{c w}{\lambda d\left(N_{w} I_{\mathrm{c}}\right)^{1 / 2}}$

where $N_{w}$ is the number of samples across the width of the line, $c$ is the speed of light, and $\lambda$ is the central wavelength of the line. For typical lines measured in our stars $(\lambda=$ $4500 \AA, w=7 \AA, N_{w}=6, d=0.2$, and $\left.I_{\mathrm{c}}=2 \times 10^{4}\right)$, we obtain $\delta v_{\mathrm{rms}}=15 \mathrm{~km} \mathrm{~s}^{-1}$. This is smaller than the velocity dispersion expected if the cluster is virialised and the total mass is close to our photometric estimate. However, the signatures of expanding stellar atmospheres and binaries may be much stronger than the virial motions. Thus, it is very important to constrain these effects with the data at hand before embarking in a high spectral resolution survey of the kinematics of the cluster.

At our resolution the Balmer lines cannot be used to measure radial velocities because they are severely contaminated by nebular emission. Therefore, we have restricted our analysis to stars with well exposed HeI and HeII absorption lines. In order to have an indication of the presence of atmospheric motions we have only considered stars with at least three He lines detected. This further restricts our sample to 97 spectra, several of which correspond to the same star.

The centroids of the lines were determined from Gaussian fits using the package ngauss within IRAF. The fitting errors were used as weights to calculate the (weighted) mean velocity of each star. A conservative $\kappa-\sigma$ filter was used to reject stars with suspected internal (atmospheric) motions. Thus, all stars with a dispersion of more than $25 \mathrm{~km} \mathrm{~s}^{-1}$ between the measured lines were rejected. The final list is presented in Table 1 that gives, for each star, the Parker number, the spectral type from Paper II, position in arcsec from the cluster center, assumed to be R136 (Selman et al. 1999a), the weighted average radial velocity, and the weighted error. A number of stars appear to be binaries on the basis of showing double peaked lines (stars \# 1024, 1369 and 1938), asymmetric line profiles (\# 222, 613, and 1191), or different radial velocities for the He I and He II lines (\# 1998). These stars tend to have larger internal errors as can be seen in the second part of Table 1.

\section{Results}

\subsection{The distribution of radial velocities}

In Fig. 1 we plot the spatial distribution of the measured stars in three different velocity bins in order to check for the presence large scale motions, such as rotation, or clustering of stars in large substructures of different kinematics. Unfortunately, the distribution of the points, dictated 
Table 1. Stellar radial velocities.

\begin{tabular}{|c|c|c|c|c|c|}
\hline Star id. & Sp.Type & $\Delta \alpha\left({ }^{\prime \prime}\right)$ & $\Delta \delta\left(^{\prime \prime}\right)$ & $\left\langle V_{\mathrm{r}}\right\rangle$ & $\sigma_{\text {int }}$ \\
\hline 15 & $\mathrm{O} 8.5 \mathrm{~V}$ & -107 & 107 & 234.9 & 14.0 \\
\hline 32 & O9 IV & -102 & 72 & 272.1 & 09.5 \\
\hline 124 & $08.5 \mathrm{~V}$ & -76 & 25 & 287.5 & 06.9 \\
\hline 316 & O6.5 V & -50 & -164 & 265.9 & 09.5 \\
\hline 541 & O7.5 V & -29 & -65 & 252.6 & 07.1 \\
\hline 649 & O8-9 V & -20 & -106 & 323.7 & 09.4 \\
\hline 713 & O5 V & -15 & -53 & 308.7 & 11.8 \\
\hline 747 & O6-8 V & -13 & -142 & 364.3 & 22.8 \\
\hline 791 & $\mathrm{O} 5 \mathrm{~V}$ & -09 & 141 & 310.7 & 08.8 \\
\hline 805 & O5-6 V & -08 & -38 & 292.1 & 09.4 \\
\hline 863 & O6.5 V & -04 & -03 & 308.0 & 06.5 \\
\hline 871 & $\mathrm{O} 4 \mathrm{~V}\left(\left(\mathrm{f}^{*}\right)\right)$ & -04 & -44 & 290.3 & 06.5 \\
\hline 901 & O3 V $\left(\left(\mathrm{f}^{*}\right)\right)$ & -02 & 26 & 276.2 & 08.7 \\
\hline 905 & O9-B0 V & -02 & 61 & 198.2 & 16.3 \\
\hline 975 & O6-7 V((f)) & 02 & -27 & 325.5 & 05.9 \\
\hline 1022 & O5: V & 04 & -139 & 320.6 & 07.2 \\
\hline 1063 & O6-7 V & 06 & 108 & 267.0 & 16.5 \\
\hline 1109 & O9 V & 09 & -167 & 229.7 & 05.9 \\
\hline 1139 & B0 V & 11 & 36 & 225.5 & 09.5 \\
\hline 1163 & O4 If: & 12 & -72 & 274.1 & 11.3 \\
\hline 1247 & B0.5 IV & 17 & 91 & 333.0 & 11.6 \\
\hline 1283 & O6 V:((f*)) & 19 & -09 & 352.2 & 06.7 \\
\hline 1339 & B0-0.2 IV & 23 & -60 & 265.5 & 12.0 \\
\hline 1389 & B1: V:: & 27 & 70 & 292.6 & 06.1 \\
\hline 1419 & B0-0.2 III-I & 31 & 98 & 259.0 & 10.0 \\
\hline 1459 & O9.5 II & 34 & 145 & 272.7 & 14.3 \\
\hline 1460 & B0-2 V & 34 & 172 & 282.7 & 21.0 \\
\hline 1468 & $09.5 \mathrm{~V}$ & 36 & 16 & 321.0 & 13.3 \\
\hline 1500 & B0.2 III & 39 & 40 & 275.2 & 09.0 \\
\hline 1531 & O6 V $((\mathrm{f}))$ & 43 & -25 & 308.0 & 09.0 \\
\hline 1553 & $\mathrm{O} 7 \mathrm{~V}$ & 47 & -09 & 321.5 & 07.3 \\
\hline 1563 & O7.5 II-III(f) & 47 & -04 & 271.6 & 06.9 \\
\hline 1584 & B0-1 V & 50 & -02 & 320.8 & 23.1 \\
\hline 1604 & B1 V & 55 & 85 & 360.2 & 17.6 \\
\hline 1614 & O5-6 V((f)) & 56 & 09 & 291.2 & 06.6 \\
\hline 1618 & B0-0.2 III & 56 & 128 & 270.1 & 07.6 \\
\hline 1619 & O8 III(f) & 56 & 102 & 357.8 & 20.0 \\
\hline 1643 & $\mathrm{O} 5 \mathrm{~V}$ & 60 & 128 & 279.0 & 06.6 \\
\hline 1661 & B1 III & 62 & 124 & 322.7 & 07.5 \\
\hline 1685 & B0.5-0.7 III-I & 66 & 161 & 291.8 & 11.6 \\
\hline 1729 & B1 II-III & 71 & 80 & 283.2 & 17.3 \\
\hline 1737 & B1.5 III & 71 & 139 & 339.1 & 05.7 \\
\hline 1969 & B0.7 IV & 113 & 74 & 329.6 & 12.7 \\
\hline 1987 & B2 I & 120 & -113 & 294.8 & 05.0 \\
\hline 10001 & $\mathrm{O} 4 \mathrm{~V}$ & & & 246.1 & 14.7 \\
\hline 10003 & B1-1.5 V & & & 279.9 & 06.9 \\
\hline \multicolumn{6}{|c|}{ Suspected Binaries } \\
\hline 222 & O9.5-B0 V & -62 & 143 & 198.9 & 26.4 \\
\hline 613 & O8.5 V & -23 & -154 & 203.2 & 13.7 \\
\hline 1024 & O9-B0 V & 05 & -110 & 510.7 & 27.9 \\
\hline 1191 & B0.2-1 III-V & 13 & -30 & 345.7 & 28.8 \\
\hline 1369 & $08.5 \mathrm{~V}$ & 26 & -09 & 318.1 & 78.5 \\
\hline 1938 & $\mathrm{O} 7.5 \mathrm{~V}$ & 107 & 134 & 350.0 & 18.0 \\
\hline 1988 & B0.5 V & 121 & -22 & 300.0 & 18.0 \\
\hline
\end{tabular}

by the geometry of the spectral masks, precludes a finer analysis, but a visual inspection of the graph reveals no evidence of clumping of stars with similar velocities, nor

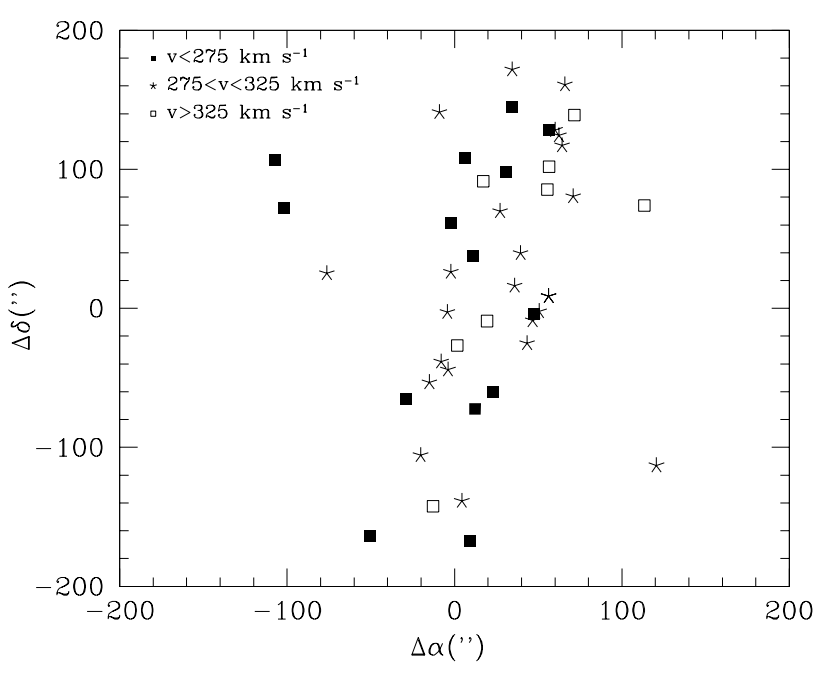

Fig. 1. Spatial distribution of stellar radial velocities. Two stars are missing, as they fall out of the photometry area.

of rotation along the axis defined by the observational technique.

We can safely proceed, therefore, to draw histograms and estimate velocity dispersions. Figure 2 presents velocity histograms of the single stars in Table 1 with two different bin sizes, and two different origins to check for sampling effect. The top two panels are for a binning of $11 \mathrm{~km} \mathrm{~s}^{-1}$ (corresponding to our estimate for the mean random error in the velocities) and the lower two for a binning of twice this error. The multiple peaks of the first plot disappear when the bins are shifted by $5 \mathrm{~km} \mathrm{~s}^{-1}$ (half a step), indicating that they are artifacts of the small number statistics. This is confirmed in the lower panel where shifting the sampling by half a step $\left(11 \mathrm{~km} \mathrm{~s}^{-1}\right)$ does not change the distribution in any significant way. The hypothesis of a Gaussian distribution is valid, based on $\chi^{2}$ tests performed to the distribution.

We conclude that there is no evidence for statistically significant peaks in the radial velocity distribution of the cluster. Thus, we can use all the data to estimate the velocity dispersion of the cluster. After correction for measurement (internal) errors and zero point errors (from the [O I] Auroral line), the radial velocity dispersion of 48 single stars in the cluster is $32 \mathrm{~km} \mathrm{~s}^{-1}$. This is much larger that the value expected if the cluster is virialised with a total mass equal to the photometric mass and also much larger than our combined errors $\left(\sigma_{\text {tot }} \sim 15 \mathrm{~km} \mathrm{~s}^{-1}\right)$.

\subsection{Binaries}

Spectroscopic binaries are very difficult to detect in a single observation, specially single-lined ones. Clearly, therefore, the number of binary candidates listed in Table 1 is only a lower limit. This is consistent with the fact that studies of young open clusters indicate $30 \%$ to be a typical percentage of binaries detected in these systems (Garmany et al. 1980; Levato et al. 1991) to be compared to $13 \%$ in our sample. 

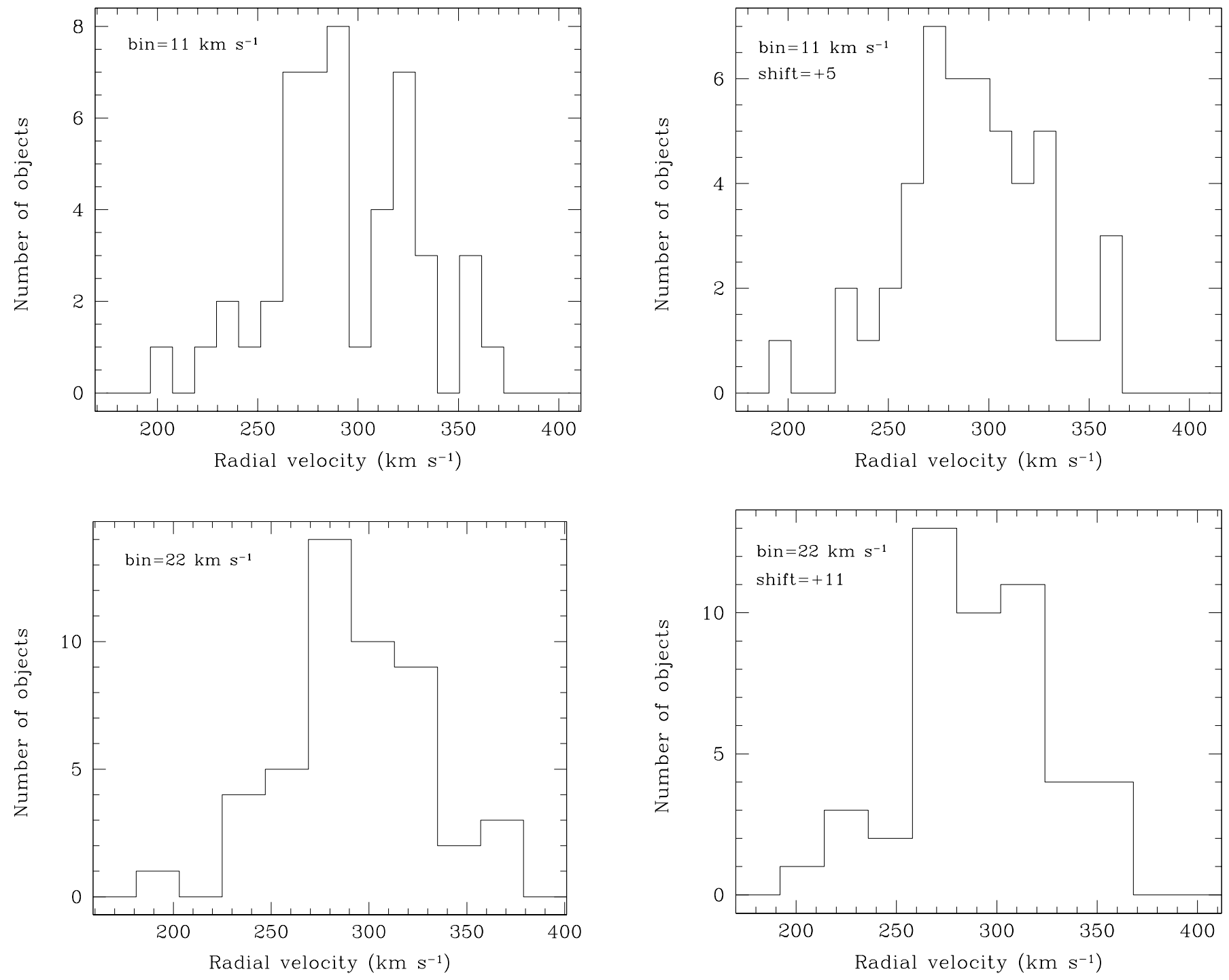

Fig. 2. Distribution of radial velocities for two bin sizes of corresponding to the typical measurement error $\left(11 \mathrm{~km} \mathrm{~s}^{-1}\right.$, top panels) and twice that error (bottom panels). The histograms are shifted by half a bin between the left and the right panels to illustrate features due to sampling statistics.

Our data alone, therefore, can only be used to determine a lower-limit to the effect of binaries in the observed velocity dispersion of NGC 2070. An upper limit can be obtained using the Montecarlo simulations of Bosch \& Meza (1998). Assuming that all the stars in the cluster are binaries with their center of mass at rest within the cluster the models predict a velocity dispersion of $\sigma_{\text {bin }} \sim 35 \mathrm{~km} \mathrm{~s}^{-1}$. This must be compared with our observed dispersion of $46.5 \mathrm{~km} \mathrm{~s}^{-1}$ including the 55 stars of Table 1 (corrected for observational errors). If we exclude star \#1024 with a radial velocity of $510 \mathrm{~km} \mathrm{~s}^{-1}$ which may not be a member of the cluster, the dispersion is reduced to $36.5 \mathrm{~km} \mathrm{~s}^{-1}$. This result is consistent, within the uncertainties, with the hypothesis that most of our observed velocity dispersion for the cluster is due to binary motions. A (very uncertain) lower limit for the virial motions of the stars in the cluster potential is thus, $\sigma_{\text {vir }}=\sqrt{36.5^{2}-35^{2}} \sim 10 \mathrm{~km} \mathrm{~s}^{-1}$.
For comparison purposes we can estimate the expected velocity dispersion assuming the cluster is virialised and the total mass is equal to the upper photometric mass limit from Paper III. From the density distribution derived in the same paper, we estimate a core radius of $0.5 \mathrm{pc}$ which yields $\sigma_{\text {phot }}=18 \mathrm{~km} \mathrm{~s}^{-1}$.

\subsection{Mass segregation}

In Paper II, using only the stars with spectroscopy, we found that the most massive stars in NGC 2070 were preferentially found closer to the center of the cluster. This was interpreted as tentative evidence in favor of the existence of mass segregation, as was originally advocated by Malumuth \& Heap (1995). This conclusion was somewhat weakened in Paper III which presented a detailed analysis of the IMF in several rings around the cluster center. 
Table 2. Mass segregation.

\begin{tabular}{rrr}
\hline \hline Mass range & Mean mass & Velocity dispersion \\
\hline$>23.5 M_{\odot}$ & $49.6 M_{\odot}$ & $27.8 \mathrm{~km} \mathrm{~s}^{-1}$ \\
$<23.5 M_{\odot}$ & $19.4 M_{\odot}$ & $36.7 \mathrm{~km} \mathrm{~s}^{-1}$ \\
\hline
\end{tabular}

The IMF was found to have the Salpeter slope almost everywhere with the exception of the very core where, combining intermediate mass HST data from Hunter et al. (1995) with our high mass end data, we found marginal evidence for flattening. The most important "mass segregation" was found in a "ring" $6 \mathrm{pc}$ away from the cluster center, again weakening the idea that closer to the center we would find the major relative concentration of massive stars. We should point out in the context that, because of the strong density gradient, the half-mass ratio of the cluster is very small. This explains the large concentration of high mass stars in the central parsec of the cluster found by Massey \& Hunter (1998).

The two-body relaxation time for NGC $2070^{1}$ is about two orders of magnitude larger than the age of the stars. Therefore, if mass segregation is indeed present it must be primordial (Bonnel \& Davies 1998; Elmegreen 2000). In either case, dynamical or primordial, we expect to see a difference in the velocity dispersion of the stars as a function of mass in the sense of it being lower for more massive stars. Moreover, if mass segregation has a dynamical origin, we expect to see energy equipartition between stars of different masses (Spitzer 1969).

We can test these hypotheses by splitting the observed non-binary stars into two equal groups of 24 objects according to their masses as indicated by their spectral types (Table 1). The result is presented in Table 2.

The Fischer F-test on both distributions gives a value of $F=1.6$ corresponding to a probability of $27 \%$ that both samples are drawn from the same parent distribution. Thus, there is tentative, but not conclusive, evidence that the massive stars have a lower dispersion. The ratio of mean energy $\left(M^{2}\right)$ between the two mass bins is $\sim 1.5 \pm 0.1$, significantly different from the equipartition ratio, $r=1$. So if the mass segregation is indeed present, it is most likely not due to two-body relaxation. We remark, however, that our radial velocity data samples very sparsely the inner $10 \mathrm{pc}$ of the cluster, where we concentrated our photometric study, and which contains most of the cluster mass.

\section{Conclusions}

In spite of the relative low spectral resolution, our data already provide important new results about the dynamical state of the 30 Dor cluster. First, the velocity dispersion is much too large to be due random motions of the stars in the gravitational potential of the cluster. Instead, the observed dispersion seems to be entirely dominated by binary orbital motions. Thus, the first important results is that higher spectral resolution alone is not sufficient to probe the dynamics of the cluster; it is also crucial to have observations with good time resolution in order to find (and exclude) binaries. Second, there is no strong evidence for dynamical mass segregation in the sense of massive stars moving with lower random velocities. If present, the effect is masked by binaries, so again, it is crucial to obtain data for several epochs. Finally, the virial dynamical mass of the cluster is comparable within factors of a few with the photometric mass. Therefore, using a reasonable number of non-binary stars it should be possible to place useful constrains on the IMF slope below $1 M_{\odot}$.

The strong conclusion of this investigation, therefore, is that it would be very worthwhile to obtain time resolved spectroscopy of a sample of 100-200 stars in the cluster. The FLAMES integral field spectrograph on the VLT appears ideally suited for such study.

\section{References}

Aller, L. H., Appenzeller, I., Baschek, B., et al. 1982, in Landolt-Bornstein: Numerical Data and Functional Relationships in Science and Technology

Bonnell, I. A., \& Davies, M. B. 1998, MNRAS, 295, 691

Bosch, G., \& Meza, A. 1998, IX Latin American Regional Meeting, Rev. Mex. Astron. Astrofis. Conf. Ser., 11, 29

Bosch, G., Terlevich, R., Melnick, J., \& Selman, F. 1999, A\&AS, 137, 21 (Paper II)

Brown, T. M. 1990, ASP Conf. Ser., 8, 335

Clarke, C. J. 2001, IAU Symp., 207, in press

Elmegreen, B. G. 2000, ApJ, 539, 342

Garmany, C. D., Conti, P. S., \& Massey, P. 1980, ApJ, 242, 1063

Hunter, D. A., Shaya, E. J., Hoiltzmann, J. A., et al. 1995, ApJ, 459, L27

Inagaki, S., \& Saslaw, W. C. 1985, ApJ, 292, 339

Levato, H., Malaroda, S., Garcia, B., et al. 1991, Ap\&SS, 183, 147

Massey, P., \& Hunter, D. A. 1998, ApJ, 493

Malumuth, E. M., \& Heap, S. R. 1995, AJ, 107, 1054

Selman, F., Melnick, J., Bosch, G., \& Terlevich, R. 1999a, A\&A, 341, 98

Selman, F., Melnick, J., Bosch, G., \& Terlevich, R. 1999b, A\&A, 347, 532 (Paper III)

Spitzer, L. 1969, ApJ, 158, L139

Walborn, N. R. 1991, The Magellanic Clouds, IAU Symp., 148, 145

\footnotetext{
1 Although for systems with a wide spectrum of masses the relaxation times are much shorter (Inagaky \& Saslaw 1985).
} 